\title{
Uncontrolled manifold and Juggling: Retrieving a set of Controlled Variables from Data
}

\author{
Vittorio Lippi $^{*} \quad$ Emanuele Ruffaldi $^{*}$ Gregory Zelic ${ }^{\dagger} \quad$ Julien Lagarde ${ }^{\dagger}$ Paolo Tripicchio \\ Carlo Alberto Avizzano* \\ (*) PERCRO Scuola Superiore SantÁnna, Italy \\ (†) UM1, France \\ E-mail:v.lippi@sssup.it,e.ruffaldi@sssup.it
}

\begin{abstract}
In this paper we analyze the concept of UnControlled Manifold (UCM), that consists in the kinematic variables that are not controlled by the user, being not relevant to the task. We proceed testing a set of controlled variables inspired by the literature about tracking task, then we propose a procedure to identify them on the basis of captured data.

We are interested in the analysis of behavior in a Virtual Environment and in the real world. In particular we analyze the three ball cascade juggling and its simulation through a platform named Light Weight Juggling focusing on the task of ball tossing.

Users arm kinematics is represented as a robotic manipulator with 7 degrees of freedom. Joint angles are retrieved through an optical tracking system. The variables controlled in the virtual environment are a subset of the ones controlled in the real world, that leads to an UM that differs from the one in the real world. A comparison between the statistics computed in the two cases is performed to explore behavioral differences in the two cases.
\end{abstract}

\section{INTRODUCTION}

We explore the behavior through the concept of UCM, the UnControlled Manifold (see section 1.2 for a detailed description), that basically represents a set of kinematics variables that are not controlled by the user being irrelevant for the task performed.

In this work, in particular, the three ball cascade juggling is analyzed. Juggling is a complex task, requiring a range of different skill components. To successfully perform different tricks, the expert juggler must integrate the multiple skill components such as bimanual coordination, procedural organization, perception by touch, eye-hand coordination, balance and postural control.

We will proceed through the following steps. First we will take in account directly the position variables affecting the tasks, taking the kernel of the Jacobian as the UCM linear approximation as done in [8] for a reaching task. Then we will try to proceed backward from joints variables variance to the definition of the controlled variables, we will use a principal component analysis to define the UCM without a prior knowledge of them.

\subsection{VIRTUAL ENVIRONMENT}

The Light Weight Juggling (LWJ) application allows the user to perform juggling (three ball cascade) in virtual reality[6]. The specific outcome of training with the juggling demonstrator is to provide a non-juggler with the skills required to successfully juggle the three ball cascade and similar level tricks.

The user interacts with the simulated environment through the Pohlemus three dimensional tracker. It is used to get the hand position in real time. It works getting the position and the orientation of two sensors linked to the user hands. The virtual environment consist of a three dimensional representation of Juggling portraying two hands controlled in position by the user and the three balls to be juggled. There is not any kind of haptic feedback. The tosses are triggered by hand acceleration and the catches are triggered by hand position.

This is an Open Access article distributed under the terms of the Creative Commons Attribution-Noncommercial License 3.0, which permits unrestricted use, distribution, and reproduction in any noncommercial medium, provided the original work is properly cited. 


\subsection{THE UNCONTROLLED MANI- FOLD}

In order to define the (UCM) [8] concept a set of variables controlled by the nervous system should be identified. These variables may be particular functions of the joint angles. In a tracking task performed by a human, where hand position is the objective, it can be assumed that the coordinates defining the position of the hands are themselves the controlled variables. In this case the UCM is the set of joint configurations keeping the hand in the considered position and orientation $P$. Notice that the controlled variables should be defined according to the task. Given the kinematic description of the human arm presented in the previous section, the vector $P$, can be expressed as function of the joint variables $q_{i}$. For a given value $P_{i}$ of the controlled variables there is a set of variables defining a surface in the space of joints configuration producing that value can be found, in the case we proposed it is the set of joint configurations keeping the hand in the considered position.

After selecting a candidate set of variables, the hypothesis is being verified by means of a comparison of the analysis of variance along the directions of the UCM and the ones orthogonal to it. The hypothesis is verified if a larger variance is obtained on the directions along the UCM. Variance is computed repeating the analyzed movement and aggregating them in specific phases of the motion itself. The UCM is in general a curved surface, hence a linearization is needed in order to compute the variance. The linearization is applied around a given point in joint configurations space $q_{m}$. Given a kinematic model of human arm we can define this approximation through the Jacobian matrix $J_{i, j}(q)=\frac{\partial P_{j}(q)}{\partial q_{i}}$ It is possible to define the linearized hand position $P^{L}$ as:

$$
P^{L}=J\left(q_{m}\right) q
$$

The UCM is then approximated locally by the kernel $K\left(J\left(q_{m}\right)\right.$ of the Jacobian in the point $q_{m}$, that is a vector subspace of joint configurations. The joint configurations affecting hand positions, the ones we assumed controlled by the nervous system, are hence approximated by $K^{\perp}\left(J\left(q_{m}\right)\right)$, the directions orthogonal to the Jacobian kernel that is equivalent to the image of the transposed Jacobian $\operatorname{Im}\left(J\left(q_{m}\right)^{T}\right)$.

\subsection{Human Arm Kinematics}

Human arm is modeled as a manipulator with seven degrees of freedom: three in the shoulder movement one in the elbow flexion and three in the wrist. This model is represented in figure 1[5]. The description in

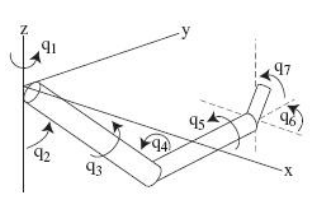

Figure 1: Model of human arm with associated joint variables, from [5]

Table 1: Human arm Denavit-Hartenberg parameters

\begin{tabular}{|c|c|c|c|c|c|}
\hline Link & Articulation & $\alpha$ & $\mathrm{A}$ & $\theta$ & $\mathrm{d}$ \\
\hline \hline 1 & Shoulder & $\pi / 2$ & 0 & 0 & 0 \\
\hline 2 & Shoulder & $\pi / 2$ & 0 & 0 & 0 \\
\hline 3 & Shoulder & $-\pi / 2$ & 0 & 0 & $L_{3}$ \\
\hline 4 & Elbow & $\pi / 2$ & $L_{2}$ & 0 & 0 \\
\hline 5 & Wrist & $-\pi / 2$ & 0 & 0 & 0 \\
\hline 6 & Wrist & $\pi / 2$ & 0 & 0 & 0 \\
\hline 7 & Wrist & 0 & 0 & 0 & $L_{3}$ \\
\hline
\end{tabular}

standard Denavit-Hartenberg convention is reported in Table 1.

\subsection{UCM in three Ball Cascade}

Several works about the UCM propose an analysis of a reaching task [9][8][10] and we may ask if the performer is controlling the position during the tossing task. We may start with the assumption we presented in the previous section taking in account the six degrees of freedom of the hand, in terms of positions and orientations as controlled variables. There are many possible ways to build the Jacobian[4], in this case we are able to compute it analytically as $J_{\text {real }}=J_{\text {arm }}$, that is directly the Jacobian of the human arm model, and $J_{L W J}$ corresponds to the rows of $J_{\text {real }}$ associated with the horizontal and the vertical movement, that, as explained in section 1.1 are the ones controlled into the virtual environment.

To evaluate the linear approximation we compute the error it produces over the position of the hand effector as expressed in eq. 2 . In figure 2 we plot this error over the displacement from the average point. To compute the variance we take in account just the samples for which the error produced is below two centimeters: most of the sample set lies below this threshold.

Identifying the UCM with the kernel space of arm's Jacobian means linearly approximating it at a point in the joint space. The first step to apply this analysis is to identify the points where to approximate it. Tossing points have been selected for this purpose. Once a 


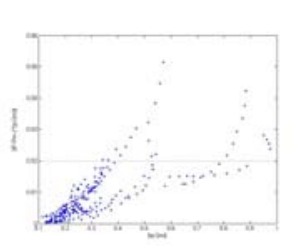

Figure 2: Position error [m] produced by the linearization plotted over the norm of the difference between the mean joint configuration and the one of each sample [rad].

Table 2: Variance per degrees of freedom

\begin{tabular}{|l|l|l|l|l|}
\hline & $K\left(J_{\text {real }}\right)$ & $K^{\perp}\left(J_{\text {real }}\right)$ & $K\left(J_{L W J}\right)$ & $K^{\perp}\left(J_{L W J}\right)$ \\
\hline Real & 0.0161 & 0.0144 & 0.0101 & 0.0427 \\
\hline LWJ & 0.0075 & 0.0068 & 0.076 & 0.0189 \\
\hline
\end{tabular}

set of tosses has been identified a mean joint configuration is found for the set of $q_{\text {toss }}$. This configuration is then used to compute the Jacobian $J\left(q_{\text {toss }}\right)$. The linear approximation could be validated, over points $q_{i}$ belonging to the considered set, computing the difference between it and the actual hand position $P$.

$$
\epsilon_{i}=P-J\left(q_{\text {toss }}\right) q_{i}
$$

The decomposition of variance for joint positions is summarized in table 2 . The distribution of variance for position does not support the assumption we made about the set of controlled variables identifying them with the position and the orientation of.

\section{Retrieving a set of Controlled Variables from Data}

Since an a priori definition of the controlled variables is not trivial in such a complex task we can reverse the process checking for directions into the joint space bearing most of the variance, then, using the Jacobian of the arm model we can check which is the corresponding set of not controlled variables. We proceed performing a principal components analysis (PCA) on the set of joint variables for both the cases. This way we find seven linearly independent variables associated with different variances. We chose a threshold of $80 \%$ of variance to determine which direction can be considered controlled and which uncontrolled. This mean that, once we ordered the principal components by variance, we take the first $\mathrm{N}$ elements covering at least the $80 \%$ of the variance. The principal components are a linear combination of the original set of joint variables, that we

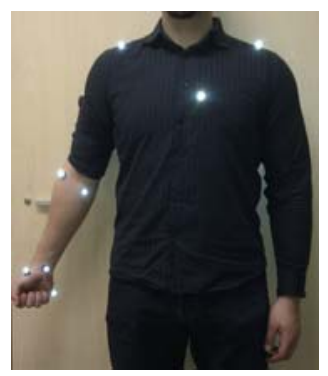

Figure 3: Configuration of markers adopted to recognize the seven degrees of freedom of the right arm. The three markers on the chest provides a reference for shoulder angles.

express through the matrix $C$. The selection of components can be expressed by the diagonal matrix $S$ having the component $S_{i, i}$ set to zero if the $i^{\text {th }}$ principal component is part of the UCM, and set to one otherwise. Then using the arm Jacobian $J_{\text {arm }}$ we may define the task related Jacobian (for the given variance threshold) as:

$$
J_{80 \%}^{t a s k}=J_{a r m} C^{-1} S C
$$

\section{Acquisition}

In order to implement the statistics described into the previous sections joint angles described in section 1.3 are needed. To acquire with precision human arm joints variables a VICON motion tracking system[1] has been used. The VICON system is based on infrared optical technology and it is suited for the acquisition of complex moving objects linked together with specific kinematic constraints. The hardware of such a system comprise several infrared cameras (in our setup a total of Mx20+ 8 cameras have been used for the acquisition) connected to a master unit module and a computer part. A specific software is then capable of recording and analyzing the data acquired from the cameras to obtain a kinematic reconstruction of the tracked subjects.

To detect tosses of the balls we covered juggling balls with reflective materials allowing to acquire the ball motion and detect the exact instant of detach from the user hand.

\section{RESULTS}

We applied the procedure described in section 1.4 to both the real juggling and the LWJ data. We set a threshold for the variance fraction of $80 \%$. Firstly we considered joints positions as kinematic variables and we assumed the controlled variables were a subspace of hand position and orientation. As result we obtained 


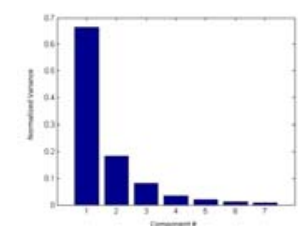

Figure 4: Variance for each principal component for real world data. The variance is expressed as fraction of the total such as it sums to one over the seven components

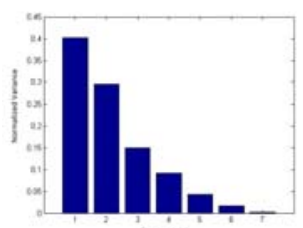

Figure 5: Variance for each principal component for real LWJ data. The variance is expressed as fraction of the total such as it sums to one over the seven components

$J_{80 \%}^{R e a l}$ of rank 5 and $J_{80 \%}^{L W J}$ of rank 4 . The different distributions along the principal components is shown in figures 4 and 5 .

\section{CONCLUSIONS AND FUTURE WORKS}

In this work we proposed to identify the controlled variables for a task starting from a PCA of joint variables. We used data from Three ball cascade Juggling as an example.Comparing the ranks of $J_{80 \%}^{R e a l}$ and $J_{80 \%}^{L W J}$ for the positions we find that the real juggling requires the user to control a space of higher dimension. This procedure finds its application in the context of digital representation of complex human skills[2], giving an indication on which variables should be taken into account to describe the task.

Our ongoing work on this topic is focused on the definition of a measure of the difference between two different experimentally retrieved UCM, this will give a tool to evaluate differences in behavior during the development of a task in different contexts (e.g. virtual reality and real world). A possible development of this work would be to apply this analysis on other tasks and their associated virtual reality training system, i.e the demonstrators we are producing within the SKILLS project to which LWJ belongs, such as the rowing[7] or industrial maintenance[3].

\section{Acknowledgments}

This work was supported by EU SKILLS Integrated Project.

\section{References}

[1] O.m.g vicon system http://www.vicon.com.

[2] C. A. Avizzano, E. Ruffaldi, and V. Lippi. Skills digital representation and storage. In International Conference on Multimodal Interfaces for Skills Transfer, 2009.

[3] U. Bockholt, S. Webel, T. Engelke, H. Graf, and J. Behr. Virtual and augmented reality training platforms for transfer of skills. 2009.

[4] S. M. S. F. de Freitas and J. P. Scholz. A comparison of methods for identifying the jacobian for uncontrolled manifold variance analysis. Journal of Biomechanics, 43(4):775 - 777, 2010.

[5] D. M. Guigon E, Baraduc P. Computational motor control: Redundancy and invariance. Journal of Neurophysiology, 97:331-347., 2007.

[6] J. Lagarde, G. Zelic, C. A. Avizzano, V. Lippi, E. Ruffaldi, H. Zalmanov, V. Erev-Yehene, N. Gillian, S. OModrain, D. Mottet, and D. Gopher. The light weight juggling training system for juggling. In International Conference on Multimodal Interfaces for Skills Transfer, 2009.

[7] E. Ruffaldi, A. Filippeschi, A. Frisoli, C. A. Avizzano, B. Bardy, and D. Gopher. Sprint: a training system for skills transfer in rowing. In T. Gutierrez and E. Sanchez, editors, SKILLSO9 International Conference on Multimodal Interfaces for Skills Transfer. Bilbao,Spain., 2009.

[8] J. P. Scholz and G. Schner. The uncontrolled manifold concept: identifying control variables for a functional task. Experimental Brain Research, 126:289 386, 1999.

[9] Y.-w. Tseng and J. P. Scholz. The effect of workspace on the use of motor abundance. Motor Control, 9:75-100, 2005.

[10] J.-F. Yang, J. Scholz, and M. Latash. The role of kinematic redundancy in adaptation of reaching. Experimental Brain Research, 176:54-69, 2007. 10.1007/s00221006-0602-8. 